\title{
Progress in Synthesis and Application of Water-soluble Fluorescent Gold Nanoparticles
}

\author{
Yuying Li \\ School of Electronics and Information Engineering, Tianjin Polytechnic University, Tianjin 300387, \\ China; \\ Lyy328546012@126.com
}

Keywords: Water-soluble fluorescent gold nanoparticles, Synthesis, Application.

\begin{abstract}
Water-soluble fluorescent gold nanoparticles have been attracting great attention due to their excellent optical, electronic, catalytic and biological application in recent years. In this paper, recent research progress in the preparation methods such as using small single molecule and macromolecule to synthesize water-soluble fluorescent gold nanoparticles are presented. The applications of water-soluble fluorescent gold nanoparticles are also reviewed in catalysis, detection of metal ions, sensors and biological applications.
\end{abstract}

\section{Introduction}

As we all know, metal cannot luminesce due to their intrinsic quality, but some researchers find that metal can luminesce if the size of the material is comparable to the Fermi wavelength of an electron ( $\sim .5 \mathrm{~nm}$ for gold and silver). Since this phenomenon was found, fluorescent metal nanoparticles have been attracting increasing attention on account of their unique optical, electronic, catalytic properties and biological application in recent years [1-2]. Among various metal nanoparticles, gold nanoparticles (GNPs) have received extensive studies for their good conductivity, surface plasmon absorption effect, small size effect, quantum size effect and excellent biocompatibility, which lead to the rapid expansion of gold nanoparticles in catalysis, detection of metal ions, sensors and biological applications $[3,4]$. Wet chemistry was used to synthesize these gold nanoparticles. The most convenient and effective method to synthesize water-soluble fluorescent nanoparticles is using reducer to make $\mathrm{Au}^{3+}$ convert to $\mathrm{Au}$, then the nanoparticles can luminesce. There are many applications for water-soluble fluorescent gold nanoparticles such as catalysis, detection of metal ions, sensors and biological applications. In this article, the research progress in synthesis and application of water-soluble fluorescent gold nanoparticles is described.

\section{Synthesis of Water-soluble Fluorescent GNPs}

In 1998, Wilcoxon et al. first found photoluminescence from gold nanostructure which can produce strong blue emission with a maximum emission wavelength at $440 \mathrm{~nm}$, the size of the nanoparticles is $5 \mathrm{~nm}$ and the quantum yield is about $10^{-5}[1]$. Since then, an increasing number of researchers began to explore various ligands to synthesize fluorescent gold nanoparticles[5].

Using monolayer. Gold nanoparticles can be synthesized in aqueous solution by a variety of different strategies. Monolayer can be chosen as the reducer and protector to synthesize fluorescent GNPs. Using smonolayer is the most simple and efficient way to synthesize fluorescent GNPs, because no more than $2 \mathrm{~h}$ and only a few steps are needed. It is easy to operate and is of great reproducibility. Glutathione[6-7], phosphine [8], sodium dodecyl sulfonate (SDS) [9], dihydrogen lipoic acid (DHLA) [10], Mercapto succinic acid (H2MSA) [11] are commonly used. The GNPs synthesized by this method is water-soluble and of good dispersity, and it can give strong luminescence. Zheng et al. successfully synthesize yellow and red emitting gold nanoparticles which give the emission at $600 \mathrm{~nm}$ using glutathione [6]. It is found that the luminescence of GNPs is owing 
to -SH in monolayer and finally -SH combine with Au to conform Au-S bond which can give strong luminescence.

Using Macromolecule. Using macromolecule as the template to synthesize and scaffold fluorescent GNPs is also available. There are four kinds of material that can be used as the template such as dendrimer, DNA, protein and peptide.

Second and fourth generation OH-terminated poly (amidoamine) PAMAM (G2-OH and G4-OH) are kind of dendrimer, which can be used to stabilize and solubilize gold nanoparticles in both aqueous and methanol solutions. Zheng's group first successfully prepared gold nanoparticles with a emission wavelength from $380 \mathrm{~nm}$ to 900nm using PAMAM in 2004[12]. The quantum yield of gold nanoparticles was improved to $42 \%$. Researchers can observe different color only by changing the ratio of PAMAM and $\mathrm{HAuCl}_{4}$. The product are all water- soluble, quantum confined and of high quantum yield. Huixiang $\mathrm{Li}$ et al. find that single- and double-stranded oligonucleotides have different propensities in absorption, and it is successfully used in colorimetric detection[13].

Using protein and peptide is a new trend to synthesize fluorescent GNPs which develops very rapidly now. The advantages in using protein and peptide as the template to synthesize gold nanoclusters are as follows. Firstly, the reaction condition is gentle and easy to realize. Secondly, the product is of excellent biocompatibility and it can be further used in biological medical diagnose and treatment. Thirdly, the product can be functionalized because protein and peptide contain functional groups which can chelate gold, such as $-\mathrm{SH},-\mathrm{NH}_{2},-\mathrm{COOH}$ etc. The most widely used protein in GNP's synthesis is bovine serum albumin (BSA). Xie et al. first synthesized gold nanoclusters with a wavelength of 640nm using BSA as reducer and stabilizer in 2009[14].The method they adopt is "one-pot" synthesis that is shown in fig.1. There are other protein which can be used as template, such as papain[15], lactoferrin[16], lysozyme [17] etc.

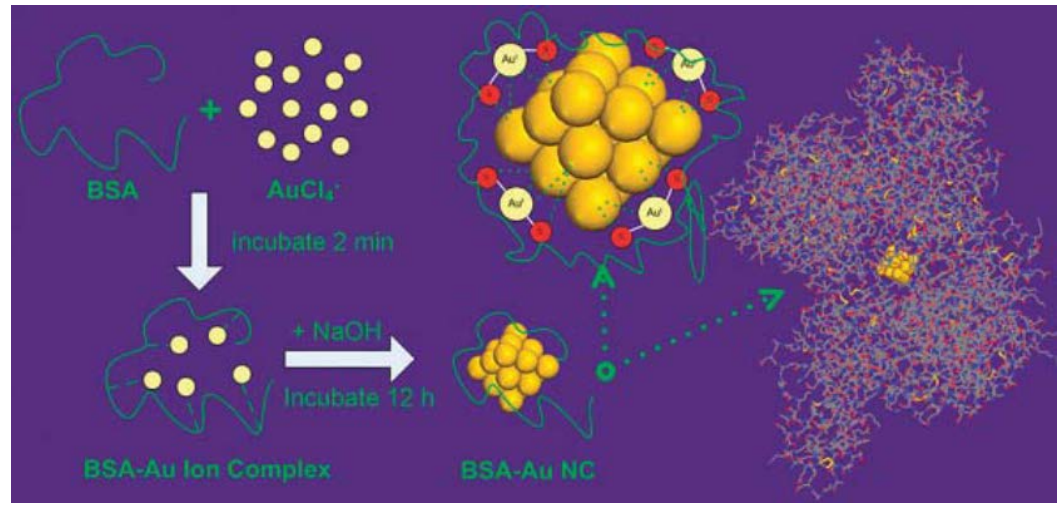

Fig. 1 Synthesis of the gold nanocluster choosing BSA as the template for the first time.

Reprinted with permission from ref 14 (Xie’ group). Copyright 2009 Journal of the American Chemical Society.

Wang et al. use the peptide sequence of CCYRGRKKRRQRR (CCYTAT) successfully synthesized gold nanoclusters with a wavelength of $677 \mathrm{~nm}$ [18]. Fig.2 shows the process of synthesizing the gold nanoclusters choosing self-designed peptide (CCYTAT) as the template.

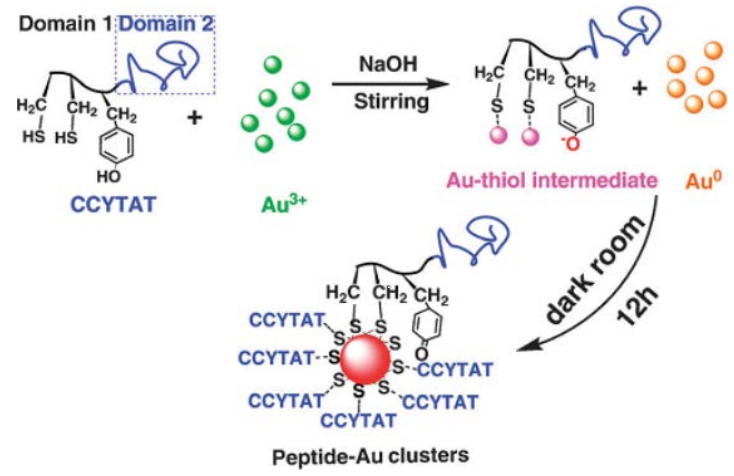


Fig. 2 Schematic illustration of synthesizing of the gold nanoclusters choosing self-designed peptide (CCYTAT) as the template. Reprinted with permission from ref 18 (Wang' group). Copyright 2012 Chemical Communications (Cambridge, England).

\section{Application of Fluorescent GNPs}

Application of water-soluble gold nanoparticles include catalysis, detection of metal ions, sensor and biological applications. Judit Oliver-Meseguer et al. have reported very small gold nanoclusters (3-10 atoms) formed from gold salt and its compound can catalyze various organic reaction at room temperature in 2012 [19]. Dingbin Liu et al. found that GNPs can detect cation $\left(\mathrm{Hg}^{2+}, \mathrm{Cu}^{2+}, \mathrm{Pb}^{2+}\right.$, $\mathrm{As}^{3+}, \mathrm{Ca}^{2+}, \mathrm{Al}^{3+}$ et al.), anion $\left(\mathrm{NO}_{2}^{-}, \mathrm{CN}^{-}, \mathrm{PF}_{6}^{-}, \mathrm{F}^{-}, \mathrm{I}^{-}\right.$et al.) and small organic molecules (cysteine, dinitrotoluene, melamine, cocaine, ATP, glucose, dopamine et al.) based on the analysis of colorimetry and fluorescence in 2011[20]. GNPs synthesized from gold salt and glutathione can be used in optoelectronic devices, many researchers have demonstrated this. Fig.3 shows a simplified scheme for the current biomedical applications of GNPs [21]. Apurba Kr. Barman et al. obtained gold nanoclusters with the emission wavelength in near infrared by using peptide polymer to replace $\mathrm{Au}_{11}\left(\mathrm{PPh}_{3}\right)_{8} \mathrm{Cl}_{3}$ and then applied in biological imaging successfully [22].

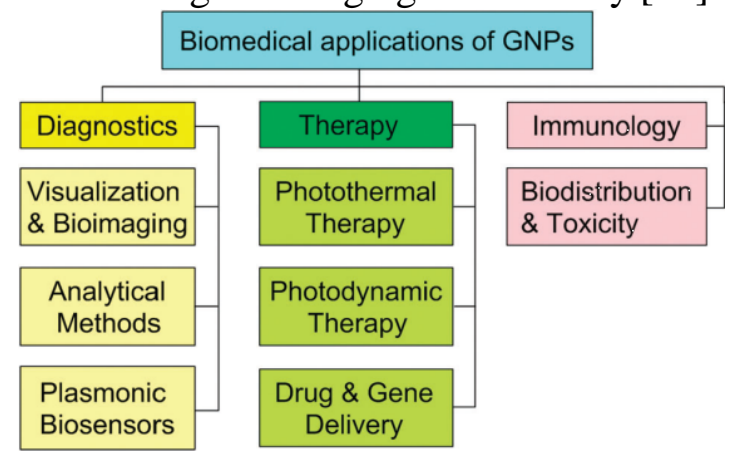

Fig. 3 Generalized scheme for the biomedical application of GNPs. Reprinted with permission from ref 21 (Lev Dykman’ group). Copyright 2011 Chemical Society Reviews.

\section{Conclusions}

An overview of the synthesis and application of water-soluble fluorescent gold nanoparticles is presented in this article. Water-soluble fluorescent gold nanoparticles is widely applied in the field of catalysis, detection of metal ions, sensor and biological applications due to its prominent characteristics and low cost, especially, GNPs revealed excellent performance in biological applications. Using monolayer is more simple and rapid in comparison with using macromolecule, but using macromolecule has more applications in biological medical diagnose and treatment than using monolayer. Functionalized GNPs can be successfully used in various cases such as diagnose, biological image and photothermal therapy. Overall, water-soluble fluorescent gold nanoparticles will play an increasing important role in the field of detection of metal ions and biological applications.

\section{References}

[1] J.P. Wilcoxon, J.E. Martin, F. Parsapour, B. Wiedenman and D.F. Kelley: Journal of Chemical Physics, Vol.108 (1998) No.21, p.9137.

[2] C. Zhou, C. Sun, M.X. Yu, Y.P. Qin, J.G. Wang, M. Kim and J. Zheng: Journal of Physical Chemistry C, Vol.114 (2010) No.17, p. 7727.

[3] P.Y. Sedeno and J.M. Pingarron: Analytical and Bioanalytical Chemistry, Vol. 382 (2005) No.4, p.884. 
[4] C.M. Welch and R.G. Compton: Analytical and Bioanalytical Chemistry, Vol. 384 (2006) No.3, p.601.

[5] W.T. Yang, W.S. Guo, B.B. Zhang, J. Chang: Acta Chimica Sinica, Vol.70 (2012). (In Chinese)

[6] J. Zheng: Fluorescent Noble Metal Nanoclusters (Ph.D., Georgia Institute of Technology, USA 2005), p.95.

[7] X. Yuan, Y. Tay, X. Dou, Z. Luo, D.T. Leong, J. Xie: Analytical Chemistry, Vol. 85 (2013) No.3, p.1913.

[8] R.C.B. Copley and D.M.P. Mingos: Journal of the Chemical Society, Dalton Transactions, Vol. 4 (1996), p.479.

[9] C.Z. Zheng, H.P. Wang, L.Z. Liu, M.J. Zhang, J.G. Liang and H.Y. Han: Journal of Analytical Methods in Chemistry, Vol. 2013 (2013) No.10, p.1155.

[10]L. Shang, L. Yang, F. Stockmar, R. Popescu, V. Trouillet, M. Bruns, D. Gerthsen, G.U. Nienhaus: Nanoscale, Vol.14 (2012) No.4, p.4155.

[11] T.U.B. Rao, T. Padeep: Angewandte Chemie International Edition, Vol.49 (2010) No.23, p.3925.

[12] J. Zheng, C.W. Zhang and R.M. Dickson: Physical Review Letters, Vol.93 (2004) No.7, p.077402.

[13] H.X. Li and L. Rothberg: Proceedings of the National Academy of the Sciences of the United States of America, Vol.101 (2004) No.39, p.14036.

[14] J. Xie, Y. Zheng, J.Y. Ying: Journal of the American Chemical Society, Vol.131 (2009) No.3, p.888.

[15] Y. Chen, Y. Wang, C.X. Wang, W.Y. Li, H.P. Zhou, H.P. Jiao, Q. Lin and C. Yu: Journal of Colloid and Interface Science, Vol.396 (2013) No.15, p.63.

[16] P.L. Xavier, K. Chaudhari, P.K. Verma, S.K. Pal and T. Pradeep: Nanoscale, Vol.2 (2010) No.15, p.2769.

[17] H. Wei, Z. Wang, J. Zhang, S. House, Y.G. Gao, L. Yang, H. Robinson, L.H. Tan, H. Xing, C. Hou, I.M. Robertson, J.M. Zuo and Y. Lu: Nature Nanotechnology, Vol.6 (2011) No.15, p.93.

[18] Y. Wang, Y. Cui, Y. Zhao, R. Liu, Z. Sun, W. Li and X. Gao: Chemical Communications (Cambridge, England), Vol.48 (2012), p.871.

[19] J.O. Meseguer, J.R.C. Antonino, I. Domínguez, A.L. Pérez and A. Corma: Science, Vol.338 (2012) No.6113, p.1452.

[20] D.B. Liu, Z. Wang and X.Y. Jiang: Nanoscale, Vol.3 (2011), p.1421.

[21] L. Dykmana and N. Khlebtsov: Chemical Society Reviews, Vol.41 (2012) No.6, p.2256.

[22] A.K. Barman, A. Chaturbedi, K. Subramaniam and S. Verma: Journal of Nanoparticle Research, Vol.15 (2013), p.2083. 